\title{
Feeding and feedback in nearby AGN - comparison with the Milky Way center
}

\section{T. Storchi-Bergmann}

\author{
Instituto de Física, Universidade Federal do Rio Grande do Sul \\ Campus do Vale, CP 15051, 91501-970 Porto Alegre RS, Brazil \\ email: thaisa@ufrgs.br
}

\begin{abstract}
I discuss feeding and feedback processes observed in the inner few hundred parsecs of nearby active galaxies using integral field spectroscopy at spatial resolutions of a few to tens of parsecs. Signatures of feedback include outflows from the nucleus with velocities ranging from 200 to $1000 \mathrm{~km} \mathrm{~s}^{-1}$, with mass outflow rates between 0.5 and a few $\mathrm{M}_{\odot} \mathrm{yr}^{-1}$. Signatures of feeding include the observation of gas inflows along nuclear spirals and filaments, with velocities ranging from 50 to $100 \mathrm{~km} \mathrm{~s}^{-1}$ and mass flow rates from 0.1 to $\sim 1 \mathrm{M}_{\odot} \mathrm{yr}^{-1}$. These rates are 2-3 orders of magnitude larger than the mass accretion rate to the supermassive black hole (SMBH). These inflows can thus lead, during less than one activity cycle, to the accumulation of enough gas in the inner few hundred parsecs, to trigger the formation of new stars, leading to the growth of the galaxy bulge. Young to intermediate age stars have indeed been found in circumnuclear rings around a number of Active Galactic Nuclei (AGN). In particular, one of these rings, with radius of $\approx 100 \mathrm{pc}$ is observed in the Seyfert 2 galaxy NGC 1068, and is associated to an off-centered molecular ring, very similar to that observed in the Milky Way $(\mathrm{MW})$. On the basis of an evolutionary scenario in which gas falling into the nuclear region triggers star formation followed by the triggering of nuclear activity, we speculate that, in the case of the MW, molecular gas has already accumulated within the inner $\approx 100 \mathrm{pc}$ to trigger the formation of new stars, as supported by the presence of blue stars close to the galactic center. A possible increase in the star-formation rate in the nuclear region will then be followed, probably tens of millions of years later, by the triggering of nuclear activity in Sgr A*.
\end{abstract}

Keywords. galaxies: active — galaxies: nuclei — supermassive black holes — mass accretion rate

\section{Introduction}

In the present paradigm of galaxy formation and evolution, supermassive black holes (hereafter SMBHs) are present in most galaxy bulges (Ferrarese \& Ford 2005). These SMBH evolve, together with the host galaxy (Di Matteo et al. 2008; Kormendy \& Ho 2013) via episodes of mass accretion to the nuclear region, which may lead both to the growth of the galaxy bulge via the formation of new stars as well as to the phenomenon of nuclear activity via mass accretion to the SMBH (feeding). The nuclear activity, once triggered, drives mechanical and radiative feedback (Hopkins et al. 2005; Di Matteo et al. 2005), which influences the evolution of their host galaxies. Feeding and feedback processes which occur in Active Galactic Nuclei (AGN) thus constrain the co-evolution of galaxies and SMBHs, but their implementation have been simplistic (e.g. Springel et al. 2005; Croton et al. 2006; Somerville et al. 2008) because they are not well constrained by observations. This is due to the fact that they occur within the inner few hundred parsecs, which cannot be spatially resolved at $z \geqslant 2$ where the co-evolution of galaxies and SMBH largely occurs. It is nearby galaxies that offer the only opportunity to test in detail the prescriptions used in models of galaxy and $\mathrm{BH}$ co-evolution. 
Constraining the processes of feeding and feedback occurring in the inner few hundred parsecs of nearby active galaxies is the goal of my research group - called AGNIFS (AGN Integral Field Spectroscopy). We have been doing this via the mapping of the gas kinematics around nearby AGN at spatial resolutions of a few to tens of parsecs, characterizing inflows and outflows. In addition, we have been able, in a few cases, to map the stellar population, looking for the contribution of recent episodes of star formation which can trace the growth of the galaxy bulge.

In the present contribution, I summarize the most recent results of our AGNIFS group, pointing out similarities to what has been observed in the nuclear region of the Milky Way, even though the $M W$ nucleus is not an $A G N$, as pointed out by Deokkeun An, in his contribution to this volume.

\section{Observations}

We have based our studies on integral field spectroscopy (IFS) at the Gemini telescopes. In the optical, we have used the Integral Field Unit of the Gemini Multi-Object Spectrograph (GMOS-IFU), which has a field-of-view of $3.5^{\prime \prime} \times 5^{\prime \prime}$ in one-slit mode or $5^{\prime \prime} \times 7^{\prime \prime}$ in two-slit mode at a sampling of 0 !' 2 and angular resolution (dictated by the seeing) of $0^{\prime \prime} 6$, on average. The resolving power is $R \approx 2500$.

In the near-infrared, we have used the Near-Infrared Integral Field Spectrograph (NIFS) together with the adaptive optics module ALTAIR (ALTtitude conjugate Adaptive optics for the InfraRed), which delivers an angular resolution of $\sim 0^{\prime \prime} 1$. The field-ofview is $3^{\prime \prime} \times 3^{\prime \prime}$ at a sampling of $0^{\prime \prime} 04 \times 0.0^{\prime \prime} 1$ and the spectral resolution is $R \approx 5300$ at the $Z, J, H$ and $K$ bands.

\section{Feeding}

There is still no consensus on the mechanisms responsible for transferring mass from galactic scales down to nuclear scales to feed the SMBH. Theoretical studies and simulations (e.g. Emsellem et al. 2006) have shown how galactic bars efficiently promote gas inflow, but which seems to stall at the Inner Lindblad Resonance (ILR), located within $\approx 1 \mathrm{kpc}$ from the nucleus forming a circumnuclear ring where star formation is triggered. Inside the inner kiloparsec, Garcia-Burillo et al. (2005) show that in some galaxies stellar gravity torques can drive gas inwards towards the nucleus. Observations of the inner kiloparsec show also nuclear bars, which can drive gas to the SMBH. Nevertheless, small-scale disks and nuclear spiral arms are observed more frequently in the inner kiloparsec of active galaxies than nuclear bars (e.g. Malkan et al. 1998; Pogge \& Martini 2002; Peeples \& Martini 2006). In particular, we, in Simões Lopes et al. (2007) found that the presence of nuclear dust structures is strongly correlated with activity in early type galaxies. The dust often exhibits a disk and/or spiral morphology, and as dust is an effective tracer of cold, molecular gas, this correlation suggests that these structures map feeding channels to the active nucleus. Recent models indeed support inflows along nuclear spiral arms in active galaxies, such as those of Maciejewski (2004), Hopkins \& Quataert (2010), Piñol-Ferrer et al. (2012).

In order to test the hypothesis that nuclear disks and spirals are indeed feeding channels to the AGN, we have been measuring the gas kinematics along these structures. I summarize below the relevant results we have observed so far.

Our first observations of non-circular motions along dusty nuclear spirals was obtained via GMOS-IFU observations of the inner kiloparsec of NGC 1097 in Fathi et al. (2006), 
which has a LINER/Seyfert 1 nucleus, by measuring the $\mathrm{H} \alpha+[\mathrm{NII}] \lambda 6584 \AA$ gas kinematics in the optical. The presence of inflows along the nuclear spirals also in molecular gas was later reported by Davies et al. (2009) and more recently via ALMA (Atacama Large Millimeter/Submillimeter Array) observations by Fathi et al. (2013). We have next found similar non-circular motions along nuclear spirals - also from $\mathrm{H} \alpha+[\mathrm{NII}]$ kinematics obtained using GMOS-IFU, around another LINER nucleus, that of NGC 6951 in Storchi-Bergmann et al. (2007).

In M 81 (LINER/Seyfert 1), using again GMOS-IFU observations, we (Schnorr Müller et al. 2011) have observed rotation in the stellar velocity field within the inner $100 \mathrm{pc}$ radius, but a totally distinct kinematics for the gas, which shows inflows along the galaxy minor axis that seem to correlate again with a nuclear spiral. More recently, observations of the inner kiloparsec of another LINER/Seyfert 1 nucleus, NGC 7213 (Schnorr Müller et al. 2013b), have shown a similar result: the ionized gas emission shows a "distorted" rotation pattern. The "distortions" in the gas velocity field are clearly correlated with a nuclear spiral seen in the structure map, and the residuals - showing mostly blueshifts in the far side of the galaxy, and redshifts in the near side, can be interpreted as inflows towards the center. We have measured the mass inflow rate at a distance of $\approx 100 \mathrm{pc}$ from the nucleus as $\approx 0.2 \mathrm{M}_{\odot} \mathrm{yr}^{-1}$. One interesting result in this case is that we could measure the mass inflow rate as a function of distance from the nucleus and the largest value is observed $\approx 400 \mathrm{pc}$ from the nucleus, decreasing inwards. This result suggests that the gas is accumulating between $100 \mathrm{pc}$ and $400 \mathrm{pc}$ from the nucleus. An speculation is that this accumulated gas may give rise to the formation of new stars in the near future.

All the above discussed cases have LINER, low-luminosity active nuclei. We find that it is indeed easier to "see" the inflows in galaxies where there is no powerful outflows, as the two may appear superimposed in the line-of-sight velocity field. More recently, we have tried to look for inflows in more luminous targets, such as NGC 2110 (Schnorr Müller et al. 2013a). In this galaxy, we could isolate the inflows because it was possible to separate the kinematics in 4 distinct components. One "cold" component, which is in rotation in the galaxy plane, shows deviation from simple circular rotation, presenting again residual blueshifts in the far side of the galaxy and redshifts in the near side (when a circular velocity field is subtracted), which can be interpreted as inflow towards the center.

The nuclear spirals we have been discussing are dusty, and should thus be associated with molecular gas. Using the instrument Gemini NIFS, we began also to study the molecular gas kinematics via observations in the $K$ band. As the spectral resolution of NIFS is almost three times higher than that of the GMOS IFU, we were able to obtain the gas kinematics using channel maps along the $\mathrm{H}_{2} \lambda 2.122 \mu \mathrm{m}$ emission line profile. The first galaxy in which we found evidence of inflows, also along nuclear spirals, was NGC 4051 (Riffel et al. 2008). We found mostly blueshifts in a spiral arm in the far side of the galaxy, and mostly redshifts in the near side, that can be interpreted as inflows towards the galaxy center if we assume again that the gas is in the plane of the galaxy.

The $\mathrm{H}_{2}$ emission in the $K$ band maps the "warm" molecular gas (temperature T $2000 \mathrm{~K}$ ) which is probably only the "skin" of a much larger cold molecular gas reservoir which emits in the millimeter wavelength range (and should thus be observable with ALMA, for example). In fact, the warm $\mathrm{H}_{2}$ mass inflow rate within the inner $100 \mathrm{pc}$ of NGC 4051 is of the order of only $10^{-5} \mathrm{M}_{\odot} \mathrm{yr}^{-1}$. But previous observations of both the warm and cold molecular gas in a sample of AGN host galaxies show typical ratios 

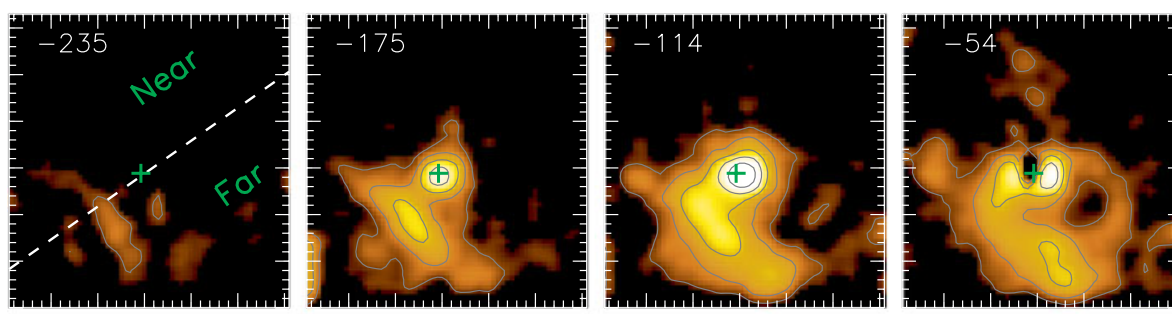

$-17.8$
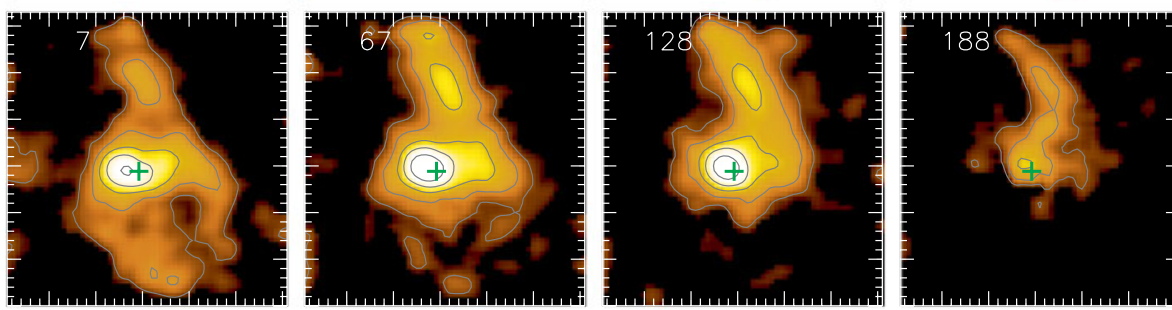

$-18.0$
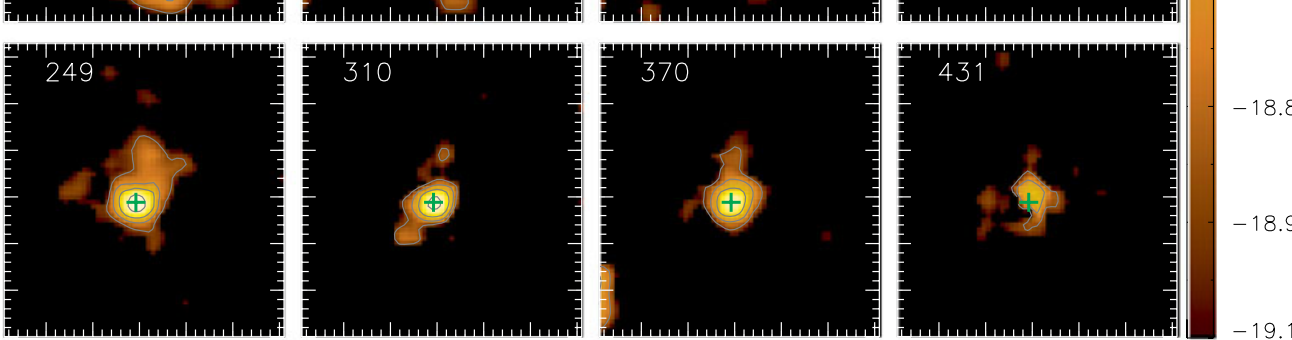

Figure 1. Channel maps of the nuclear region of Mrk 79 along the $\mathrm{H}_{2} \lambda 2.122 \mu \mathrm{m}$ emission line profile and centered at the velocity shown in the top-left corner of each panel, for a velocity bin of $60 \mathrm{~km} \mathrm{~s}^{-1}$. The dashed line shows the major axis of the galaxy, and the near and far sides of the galaxy plane are identified. The large tick marks are separated by $1^{\prime \prime}$ ( $455 \mathrm{pc}$ at the galaxy) and the angular resolution of the data is $0.12(\approx 50 \mathrm{pc})$. [A COLOR VERSION IS AVAILABLE ONLINE.]

cold/warm $\mathrm{H}_{2}$ masses ranging between $10^{5}$ and $10^{7}$ as obtained by Dale et al. (2005). Applying this ratio to NGC 4051 leads to a total gas mass inflow rate of $\geqslant 1 \mathrm{M}_{\odot} \mathrm{yr}^{-1}$.

Similar inflows in warm $\mathrm{H}_{2}$ gas were observed in the inner $350 \mathrm{pc}$ radius of the Seyfert 2 galaxy Mrk 1066 at 35 pc spatial resolution (Riffel et al. 2011a) along spiral arms which seem to feed a compact rotating disk with a $70 \mathrm{pc}$ radius. The mass of warm $\mathrm{H}_{2}$ gas is estimated as $3300 \mathrm{M}_{\odot}$ that, corrected to account for the cold component, would imply a reservoir of at least $10^{8} \mathrm{M}_{\odot}$ of cold molecular gas. The corrected mass inflow rate is $0.6 \mathrm{M}_{\odot} \mathrm{yr}^{-1}$. More recently, in Riffel et al. (2013) we have observed inflows also along nuclear spiral arms in the inner 500 pc of the Seyfert 1 galaxy Mrk 79, as illustrated in Figure 1. These channel maps show the flux distributions at the velocities indicated in each panel. The dashed line in the figure shows the major axis of the galaxy, where the near and far side of the galaxy are also identified. We found blueshifts along the spiral arm in the far side and similar redshifts in the near side supporting inflow towards the center.

\subsection{Comparison with the Milky Way (MW)}

In Simões Lopes et al. (2007) we found a strong correlation between the presence of nuclear dusty spirals and nuclear activity. The MW nucleus cannot be considered active presently; thus we would not expect to see a nuclear dusty spiral. Yet, within the inner 3 pc of the MW, a small three-armed spiral is seen, as discussed by Lacy in this volume. I speculate that - in analogy to what we have found for nearby active galaxies - that these nuclear spirals in the MW indicate that very mild inflows along these spirals could 
be occurring, leading to a very weak LINER, whose mild activity we see only because we are so close. Lacy estimates an inflow rate lower than $10^{-3} \mathrm{M}_{\odot} \mathrm{yr}^{-1}$ and point out that the spiral is co-planar to the so-called molecular circumnuclear disk (CND), argued by Liu et al. (2012) to be a possible source of material to feed gas towards the center.

\section{Feedback}

The clearest signatures of feedback from AGN are the usually collimated outflows observed in radio (the radio jets) or the broader outflows observed in emission-line kinematics of the the narrow-line region (NLR) as in Storchi-Bergmann et al. (1992), Schmitt et al. (1994), Das et al. (2006), Barbosa et al. (2009).

In the near-IR, while the $\mathrm{H}_{2}$ gas kinematics is dominated by circular rotation and/or inflows in the plane of the galaxy (at velocities of $50-100 \mathrm{~km} \mathrm{~s}^{-1}$ ), the ionized gas emission usually shows outflows (velocities of up to $1000 \mathrm{~km} \mathrm{~s}^{-1}$ ) combined with circular rotation (with typical velocities of $200 \mathrm{~km} \mathrm{~s}^{-1}$ ). The $\mathrm{H}^{+}$line-emitting gas (such as in $\mathrm{Pa} \beta$ ) is frequently dominated by rotation, while the [FeII $] \lambda 1.644 \mu \mathrm{m}$ and [FeII $] \lambda 1.257 \mu \mathrm{m}$ emitting gas is frequently dominated by the outflows. We have thus concluded that the best emission lines to trace outflows are those of [FeII].

NIFS observations of the inner $560 \mathrm{pc} \times 200 \mathrm{pc}$ of the Seyfert 1.5 galaxy NGC 4151 at $\approx 7$ pc spatial resolution in Storchi-Bergmann et al. (2010) show that, while the $\mathrm{H}_{2}$ gas is in rotation with velocities lower than $100 \mathrm{~km} \mathrm{~s}^{-1}$ within $\approx 50 \mathrm{pc}$ from the nucleus, the $[\mathrm{FeII}] \lambda 1.644 \mu \mathrm{m}$ emitting gas shows a hollow "conical" outflow with velocities of up to $800 \mathrm{~km} \mathrm{~s}^{-1}$. From the observed velocities and inferred geometry, we were able to estimate the mass outflow rate: $\approx 2 \mathrm{M}_{\odot} \mathrm{yr}^{-1}$. We are also able to obtain the kinetic power of the outflow which is only $\approx 0.3 \%$ of the bolometric luminosity of the AGN.

In the Seyfert 2 galaxy Mrk 1066, we (Riffel et al. 2001a) find that the [FeII] $\lambda 1.644$ $\mu \mathrm{m}$ emitting gas is collimated along a nuclear radio jet and reaches outflow velocities of up to $500 \mathrm{~km} \mathrm{~s}^{-1}$. From the velocity field and geometry of the outflow we estimate a mass outflow rate in ionized gas of $\approx 0.5 \mathrm{M}_{\odot} \mathrm{yr}^{-1}$, a value which is curiously of the same order as that of the mass inflow rate in $\mathrm{H}_{2}$ in this galaxy. In a similar study of the Seyfert 2 galaxy Mrk 1157, we (Riffel et al. 2011b) have obtained a mass outflow rate of $\approx 8 \mathrm{M}_{\odot} \mathrm{yr}^{-1}$, while in Mrk 79, we (Riffel et al. 2013) have obtained a mass outflow rate of $\approx 4 \mathrm{M}_{\odot} \mathrm{yr}^{-1}$.

Recently, we have used NIFS observations of the "prototypical" Seyfert 2 galaxy NGC 1068 to map the kinematics of the different gas phases, in Barbosa et al. (2014). In Figure 2 we show channel maps of the inner $200 \mathrm{pc}$ radius in the [FeII] $\lambda 1.64 \mu \mathrm{m}$ (in red) and $\mathrm{H}_{2} \lambda 2.122 \mu \mathrm{m}$ (in green) emission lines, at a spatial resolution of $7 \mathrm{pc}$. The total flux distribution in [FeII] emission shows an hourglass structure, while in the channel maps of Figure 2 it shows an " $\alpha$-shaped" structure in the blueshifted channels and a "fan-shaped" structure in the redshifted channels. We attribute the blueshifted emission to the front part of the gas outflow modeled by Das et al. (2006), while the redshifted emission is attributed to the back part of the outflow. We note that the fan-shaped structure is very similar to that observed in Planetary Nebulae (e.g. NGC 6302), suggesting a similar mechanism for the origin of the outflow. Using the inferred geometry and velocity field, we calculate a mass outflow rate of $6 \mathrm{M}_{\odot} \mathrm{yr}^{-1}$.

Figure 2 also shows that the $\mathrm{H}_{2}$ flux distribution is completely distinct from that of [FeII], as observed in previous cases, presenting a ring-like (radius $\approx 100 \mathrm{pc}$ ) morphology. The $\mathrm{H}_{2}$ kinematics shows again much smaller velocities than those observed in [FeII] and in common with other active galaxies shows also rotation. Detailed analysis of the $\mathrm{H}_{2}$ kinematics show in addition expansion in the ring in the plane of the galaxy: Figure 2 


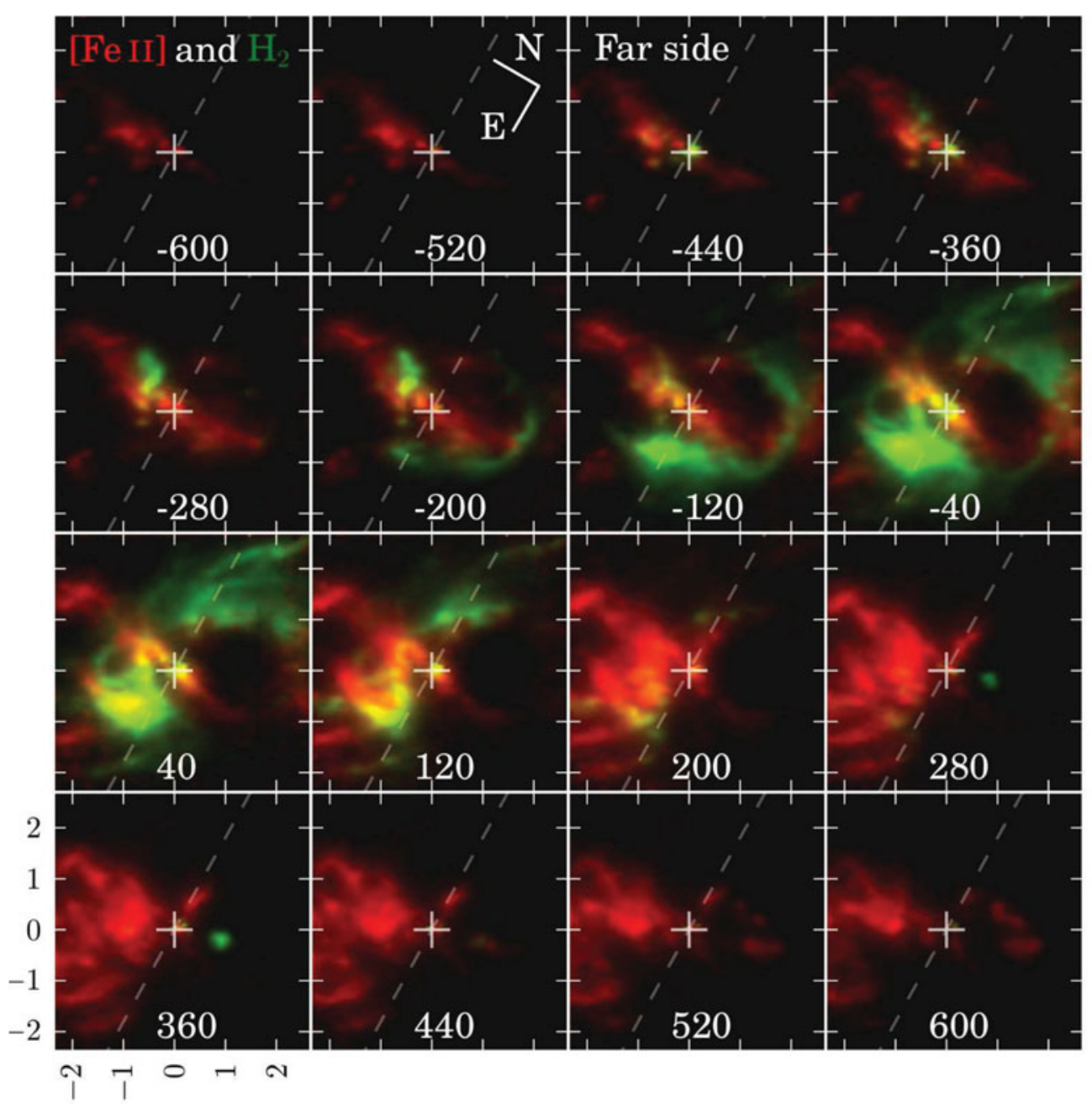

Figure 2. Channel maps of the inner $200 \mathrm{pc}$ (radius) of NGC 1068 in the [FeII] $\lambda 1.644 \mu \mathrm{m}$ (red) and in the $\mathrm{H}_{2} \lambda 2.122 \mu \mathrm{m}$ (green) emission lines. The numbers correspond to the central channel velocities in $\mathrm{km} \mathrm{s}^{-1}$. The nucleus is identified by a cross while the galaxy major axis is identified by a dashed line. [A COLOR VERSION IS AVAILABLE ONLINE.]

shows that the $\mathrm{H}_{2}$ emission is blueshifted in the near side of the galaxy and redshifted in the far side. If the gas is in the plane of the galaxy, this implies expansion of the ring. A detailed analysis of this kinematics has revealed in addition, that the expansion is decelerated from the inner towards the outer border of the ring.

\subsection{Comparison with the $M W$}

The contribution by McClure-Griffiths in these proceedings reports outflows from the MW center observed in atomic hydrogen (HI) at typical velocities of $\sim 200 \mathrm{~km} \mathrm{~s}^{-1}$. These outflows are not attributed to nuclear activity in the MW center but to winds from young stars formed in the $100 \mathrm{pc}$ ring (see discussion below), which has an estimated star-formation rate of $\approx 0.1 \mathrm{M}_{\odot} \mathrm{yr}^{-1}$.

Another signature of feedback from the MW nucleus are the so-called Fermi bubbles that extend to $\approx 20 \mathrm{kpc}$ from the nucleus. Their origin is attributed to an accretion event onto Sgr $\mathrm{A}^{*}$ or a starburst that occurred there a few $10^{6}$ yr ago, as discussed by 

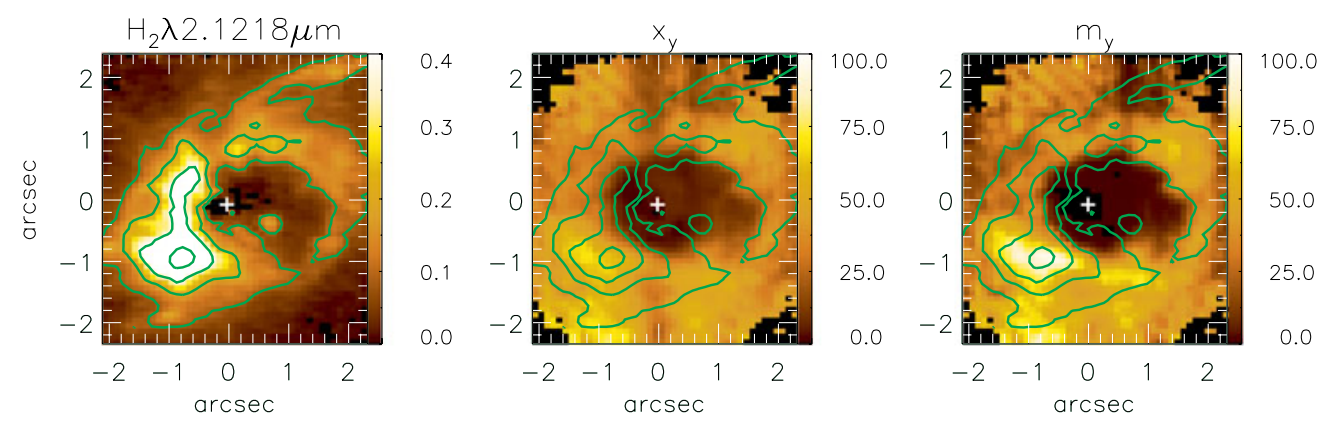

Figure 3. Left panel: $\mathrm{H}_{2}$ flux map of the inner $200 \mathrm{pc}$ radius of the galaxy NGC 1068. Central panel: contours of the $\mathrm{H}_{2}$ flux map overplotted on the map of the percent contribution of the $30 \mathrm{Myr}$ age stellar population to the total light at $2.1 \mu \mathrm{m}$. Right panel: percent contribution of the $30 \mathrm{Myr}$ age component to the total stellar mass. From Storchi-Bergmann et al. (2012). [A COLOR VERSION IS AVAILABLE ONLINE.]

Finkbeiner, Conti, Ruszkowski, and Lacki, in this volume. I note that the shape of the bubbles at their "base" (close to the nucleus) are similar to that of the outflow seen in [FeII] emission in NGC 1068 (Figure 2).

\section{Stellar population}

Using the NIFS observations of Mrk 1066, we Riffel et al. (2010) published the first resolved two-dimensional stellar population study of an AGN host in the near-IR. Using spectral synthesis, we mapped a 300 pc circumnuclear ring of intermediate age (500 Myr) stellar population which is correlated with a ring of low stellar velocity dispersion. This result has been interpreted as due to an event which led to the capture of gas to the nuclear region with enough gas mass to trigger the formation of new stars $500 \mathrm{Myr}$ ago. The low velocity dispersion indicates that these stars still keep the "cold" kinematics of the gas from which they were formed. In Riffel et al. (2011c), a similar study revealed almost the same result for the Seyfert 2 galaxy, Mrk 1157: another nuclear ring with low stellar velocity dispersion associated to intermediate age stars. Low stellar velocity dispersion rings were also found in a study of the gas and stellar kinematics of the inner kiloparsec of six nearby Seyfert galaxies (Barbosa et al. 2006 using the GMOS IFU, indicating also the presence of stars with "cold" kinematics, probably also associated with intermediate age stars.

More recently, in Storchi-Bergmann et al. (2012), we find a smaller (100 pc radius) ring of young stars in NGC 1068, dominated by ages of $\approx 30 \mathrm{Myr}$, as illustrated in Figure 3 . This ring seems to be correlated with the molecular $\left(\mathrm{H}_{2}\right)$ ring described above, as shown by the green contours in the figure, suggesting that the young stars have formed from the gas in the ring.

\subsection{Comparison with the $M W$}

Using the Herschel satellite, Molinari et al. (2011) discovered an off-centered dense elliptical ring around the MW center, with a semi-major axis of $\approx 100 \mathrm{pc}$ (see also the contribution by John Bally to this volume). We show in the right panel of Figure 4 a sketch of this ring - from the work of Molinari et al. (2011), together with an image of the NGC 1068 molecular ring observed with NIFS in the $\mathrm{H}_{2} \lambda 2.122 \mu \mathrm{m}$ emission line. Although the molecular ring in NGC 1068 seems somewhat rounder than that in the MW, it is also off-centered relative to the nucleus and has a $100 \mathrm{pc}$ radius, as in the MW. 


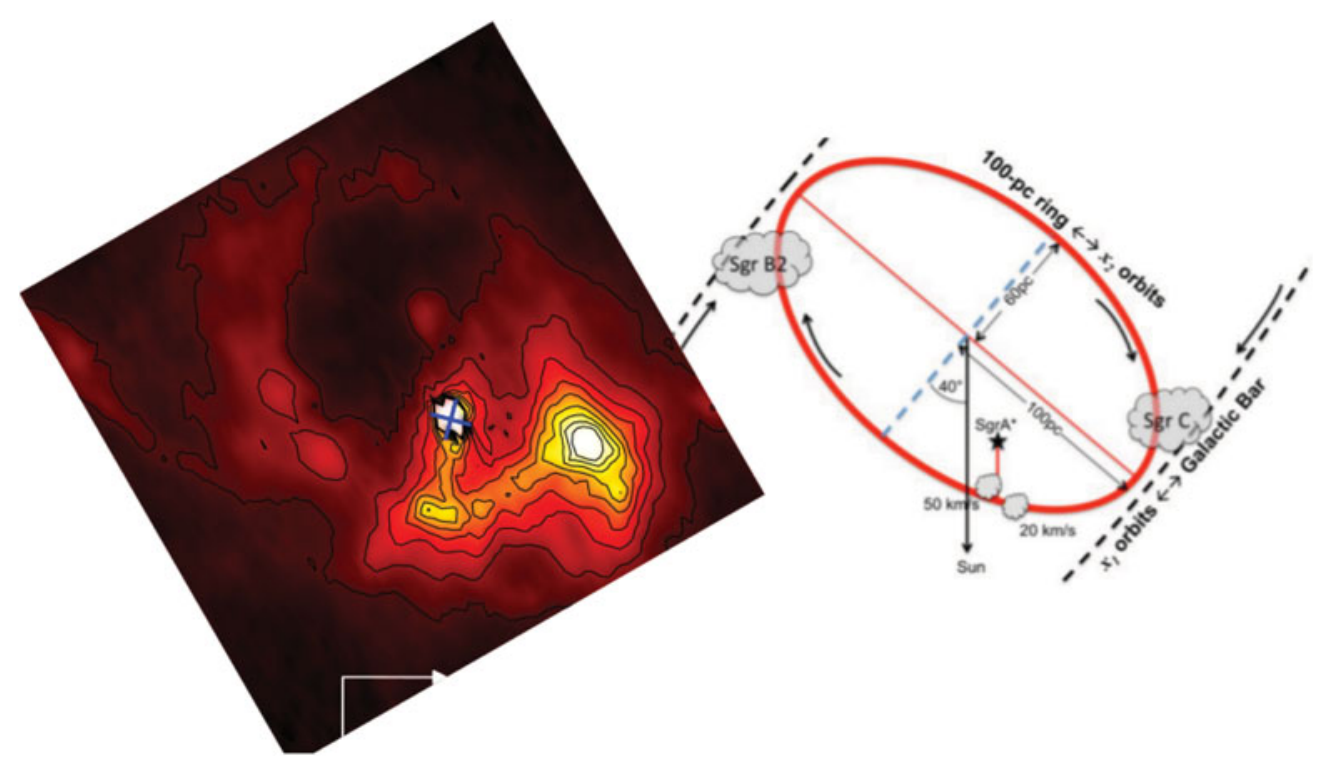

Figure 4. Two off-centered 100 pc molecular rings. Left panel: the $\mathrm{H}_{2}$ ring in NGC 1068, observed in the near-IR, from Storchi-Bergmann et al. (2012). Right panel: an sketch of the molecular ring discovered by Molinari et al. (2011) in Herschel observations of the MW central region. [A COLOR VERSion is AVAilable ONLINE.]

It has been pointed out in this Conference (e.g. in the contributions by Gallagher, Longmore, Sakamoto), that the observed star formation rate in the central region of the galaxy is too small for the amount of gas available. We speculate that it may be that the gas in the ring has been accumulating in the ring and has just begun to form stars, and that the star formation rate will increase in the near future in this ring.

\section{Summary and conclusions}

Feeding: Our observations of the kinematics of both ionized and warm molecular gas in the inner few $100 \mathrm{pc}$ of nearby AGNs reveal rotation in compact disks and inflows along nuclear spirals, with velocities in the range $50-100 \mathrm{~km} \mathrm{~s}^{-1}$ and mass flow rates from 0.01 to $\sim 1 \mathrm{M}_{\odot} \mathrm{yr}^{-1}$, observed mainly in LINERs. In Simões Lopes et al. (2007) we show a clear dichotomy between the nuclear region of early-type AGN hosts - which always show excess of dust - and that of non-AGN, supporting the hypothesis that the nuclear spirals and filaments are a necessary condition for the presence of nuclear activity. Nevertheless, the mass accretion rate necessary to feed the AGN - of $\approx 10^{-3} \mathrm{M}_{\odot} \mathrm{yr}^{-1}$, is much smaller than the above mass inflow rate. At $1 \mathrm{M}_{\odot} \mathrm{yr}^{-1}$, in $10^{8} \mathrm{yr}$ (estimated duration of an activity cycle), $\approx 10^{8} \mathrm{M}_{\odot}$ of gas will be accumulated in the inner few hundred parsecs. This mass is in agreement with that derived by Martini et al. (2013), in a recent study in which we have used Spitzer photometry of the Simões Lopes et al. (2007) sample to obtain dust masses of the nuclear spirals. The accumulation of this mass in the nuclear region will probably lead in the near future to the formation of new stars in the galaxy bulge.

Stellar population: Signatures of recent star formation in the bulge of AGN hosts have indeed been seen, in the form of rings at $100 \mathrm{pc}$ scales with significant contribution from stars of ages in the range $30 \mathrm{Myr} \leqslant$ age $\leqslant 700 \mathrm{Myr}$. These results suggest that we are witnessing the co-evolution of the SMBH and their host galaxies in the near Universe: while 
the SMBH at the center grows at typical rates of $10^{-3} \mathrm{M}_{\odot} \mathrm{yr}^{-1}$, the bulge growths at typical rates of $0.1-1 \mathrm{M}_{\odot} \mathrm{yr}^{-1}$. A similar evolution scenario has been previously proposed by Storchi-Bergmann et al. (2001).

Feedback: Our IFS observations usually reveal ionized gas outflows in nearby AGN, not so frequently in LINERS, but always observed in Seyfert galaxies. They extend to a few hundred of parsecs from the nucleus, are oriented at random angles to the galaxy plane, and reach velocities in the range $200-1000 \mathrm{~km} \mathrm{~s}^{-1}$. The total ionized gas mass in these outflows are $\sim 10^{6-7} \mathrm{M}_{\odot}$ and the mass outflow rates are in the range $0.5-10 \mathrm{M}_{\odot} \mathrm{yr}^{-1}$, which is similar to the range of the mass inflow rates, although only in a couple of galaxies we observe both inflows and outflows (e.g. in Mrk 1066). The fact that the mass outflow rates are $100-1000$ times the AGN accretion rate supports the idea that the observed outflows are due to mass loading of an AGN outflow (which should be at most equal to the AGN accretion rate) as it moves through the circumnuclear interstellar medium of the host galaxy.

Comparison with the $M W$ : Our galaxy does not have an active nucleus. Its very mild nuclear activity can only be observed because the MW nucleus is about 1000 times closer than the closest AGNs. Yet, there are some similarities with what we see around nearby AGNs: the presence of a compact molecular disk and nuclear spiral; the presence of a molecular ring at $100 \mathrm{pc}$ from the nucleus, and the presence of past energetic outflows that can be attributed to a past ejection event associated to Sgr A*.

In order to connect episodes of nuclear activity in nearby galaxies and the non-active phase of the MW, I shall use a speculative but educated evolutionary scenario that I proposed some time ago in Storchi-Bergmann et al. (2001), as follows. The nuclear activity is triggered by mass inflows towards the central region of the galaxies. The observed mass inflow rate we have measured in LINERs (of the order of one to a few $\mathrm{M}_{\odot} \mathrm{yr}^{-1}$ ) are much larger than the accretion rate necessary to power the AGN. This gas is thus probably accumulated in the inner few hundred pcs giving origin to the observed nuclear disks and spirals in the hosts, and are probably later consumed by episodes of star formation. These episodes are followed by the increase of the nuclear activity. This evolution is supported by the fact that, in the majority of active galaxies, the circumnuclear rings are dominated by stars of intermediate age $\left(\sim 10^{7-8}\right.$ yr $)$ rather than young $\left(\sim 10^{6} \mathrm{yr}\right)$, consistent with the idea that, in most AGN the nuclear activity is observed after the episodes of star formation. In this scenario, the MW, which has a molecular ring at $100 \mathrm{pc}$ and the CND within the inner $3 \mathrm{pc}$, as well as a population of young stars in the nuclear region, may have just begun the phase of starburst activity in the nuclear region, which will be followed, probably tens of millions of years into the future, by the triggering of nuclear activity in $\operatorname{Sgr} \mathrm{A}^{*}$.

\section{References}

Barbosa, F. K. B. et al. 2006, MNRAS, 371, 170

Barbosa, F. K. B., et al. 2009, MNRAS, 396, 2

Barbosa, F. K. B., et al. 2014, MNRAS, submitted

Crenshaw, D. M. \& Kraemer, S. B. 2007, ApJ, 659, 250

Croton D., et al. 2006, MNRAS, 365, 11

Das V., Crenshaw D. M., Kraemer S. B., Deo R. P., 2006, AJ, 132, 620

Dale, D. A. et al. 2005, ApJ, 129, 2197

Davies, R. I., et al. 2009, ApJ, 702, 114

Di Matteo, T., Springel, V., \& Hernquist, L. 2005, Nature, 433, 604

Di Matteo, T, Colberg, J., Springel, V., Hernquist, L., \& Sijacki, D. 2008, ApJ, 676, 33 
Emsellem, E. et al. 2006, MNRAS, 365, 367

Fathi, K., et al. 2006, ApJ, 641, L25

Fathi, K. et al. 2013, ApJ, 770, L27

Ferrarese, L. \& Ford, H. 2005, SSRv, 116, 523

Garcia-Burillo, S. et al. 2005, A\&A, 441, 1011

Hopkins, P. F., et al. 2005, ApJ, 630, 705

Hopkins, P. \& Quataert, E., 2010, MNRAS, 407, 1529

Kormendy, J. \& Ho, L. 2013, ARA\&A, 51, 511

Liu, H. B. et al. 2012, ApJ, 756, 195

Maciejewski, W. 2004, MNRAS, 354, 892

Malkan, M. A. et al. 1998, ApJS, 117, 25

Martini, P. \& Pogge, R. W. 1999, AJ, 118, 2646

Martini, P., Regan, M. W., Mulchaey, J. S., \& Pogge, R. W. 2003, ApJ, 589, 774

Martini, P., Dicken, D \& Storchi-Bergmann, T. 2013, ApJ, 766, 121

Molinari, S. et al. 2011, ApJ, 735, L33

Peeples, M. S. \& Martini, P. 2006, ApJ, 652, 1097

Piñol-Ferrer, Lindblad, Fathi 2012, MNRAS, 421, 1089

Pogge, R. W. \& Martini, P. 2002, ApJ, 569, 624

Riffel, R. A., et al. 2008, MNRAS, 385, 1129

Riffel, R. A., Storchi-Bergmann, T., Riffel, R., \& Pastoriza, M. G., 2010, ApJ, 713, 469

Riffel, R. \& Storchi-Bergmann, T. 2011, MNRAS, 411, 469

Riffel, R. A. \& Storchi-Bergmann, T. 2011, MNRAS, 417, 2752

Riffel, R., Riffel, Rogemar A., Ferrari, F., \& Storchi-Bergmann, T., 2011, MNRAS, 416, 493

Riffel et al. 2013, MNRAS, 430, 2249

Schmitt, H. R., Storchi-Bergmann, T., \& Baldwin, Jack A. 1994, ApJ, 423, 237

Schnorr Müller et al. 2011, MNRAS, 413, 149

Schnorr Müller et al. 2013a, MNRAS, in press

Schnorr Müller et al. 2013a, MNRAS, submitted

Simões Lopes, R., Storchi-Bergmann, T., Saraiva, M. F., \& Martini, P. 2007, ApJ, 655, 718

Somerville, R. et al. 2008, MNRAS, 391, 481

Springel, V. et al. 2005, MNRAS, 361, 776

Steiner, J. E., Menezes, R. B., Ricci, T. V., \& Oliveira, A. S. 2009, MNRAS, 395, 64

Storchi-Bergmann, T., Wilson, A. S., \& Baldwin, J. A. 1992, ApJ, 396, 45

Storchi-Bergmann, T., González Delgado, R. M., Schmitt, H. R., Cid Fernandes, R., \& Heckman, T., 2001, ApJ, 559, 147

Storchi-Bergmann, T., et al. 2007, ApJ, 670, 959

Storchi-Bergmann, T., et al. 2010, MNRAS, 2010, MNRAS, 402, 819

Storchi-Bergmann, T. et al. 2012, ApJ, 755, 87

Veilleux, S., Cecil, G., \& Bland-Hawthorn, J. 2005, ARAA, 43, 769 